\title{
Three-dimensional numerical simulation of nonlinear acoustic-gravity wave propagation from the troposphere to the thermosphere
}

\author{
Nikolai M Gavrilov ${ }^{1 *}$ and Sergey P Kshevetskii ${ }^{2^{*}}$
}

\begin{abstract}
Three-dimensional nonlinear breaking acoustic-gravity waves (AGWs) propagating from the Earth's surface to the upper atmosphere are simulated numerically. Horizontally moving periodical structures of vertical velocity on the Earth's surface are used as AGW sources in the model. The 3D algorithm for hydrodynamic equation solution uses finite-difference analogues of basic conservation laws. This approach allows us to select physically correct generalized wave solutions of hydrodynamic equations. The numerical simulation covers altitudes from the ground up to $500 \mathrm{~km}$. Vertical profiles of the mean temperature, density, molecular viscosity, and thermal conductivity are specified from standard models of the atmosphere. Atmospheric waves in a few minutes can propagate to high altitudes above $100 \mathrm{~km}$ after activation of the surface wave forcing. Surfaces of constant phases are quasi-vertical first, and then become inclined to the horizon below about $100 \mathrm{~km}$ after some transition time interval. Vertical wavelengths decrease with time and tend to theoretically predicted values after times longer than several periods of the wave forcing. Decrease in vertical wavelengths and increase in AGW amplitudes can lead to wave instabilities, accelerations of the mean flow and wave-induced jet streams at altitudes above $100 \mathrm{~km}$. AGWs may transport amplitude modulation of atmospheric wave sources in horizontal directions up to very high levels. Low wave amplitudes in the beginning of transition processes after activation of atmospheric wave sources could be additional reasons for slower amplitude grows with height compared to the nondissipative exponential growth predicted for stationary linear AGWs. Production of wave-induced mean jets and their superposition with nonlinear unstable dissipative AGWs can produce strong narrow peaks of horizontal speed in the upper atmosphere. This may increase the role of transient nonstationary waves in effective energy transport and variations of atmospheric parameters and gas admixtures in a broad altitude range.
\end{abstract}

\section{Findings Introduction}

Acoustic-gravity waves (AGWs) generated at tropospheric heights are frequently observed in the upper atmosphere (Fritts and Alexander 2003; Suzuki et al. 2007; Shiokawa et al. 2009; Ogawa et al. 2009, 2013). Propagating upward, AGWs can become unstable and produce turbulence and irregularities in the upper atmosphere. AGW generation may be caused, for example, by convection and mesoscale turbulence in the troposphere (e.g., Fritts and Alexander 2003; Fritts et al. 2006). These sources may have maxima at altitudes 9 to $12 \mathrm{~km}$ in the regions of tropospheric jet

\footnotetext{
* Correspondence: n.gavrilov@spbu.ru; renger@mail.ru

${ }^{1}$ Atmospheric Physics Department, Saint-Petersburg State University, 1 Ulyanovskaya St., Saint-Petersburg 198504, Russia

${ }^{2}$ Theoretical Physics Department, Immanuel Kant Baltic Federal University, Kaliningrad, Russia
}

streams (Medvedev and Gavrilov 1995; Gavrilov and Fukao 1999; Gavrilov 2007).

Most analytical and numerical models of atmospheric AGWs use linearized hydrodynamic equations. Baker and Schubert (2000) simulated behavior of nonlinear AGWs in the atmosphere of Venus. They considered wave propagation in a rectangular region of the atmosphere having vertical and horizontal sizes of 48 and $120 \mathrm{~km}$, respectively. Some authors (Fritts and Garten 1996; Andreassen et al. 1998; Fritts et al. 2009, 2011) simulated turbulence produced by breaking atmospheric waves and Kelvin-Helmholtz instabilities. Their models were three-dimensional and described waves and turbulence in rectangular boxes having limited horizontal and vertical sizes. The authors used Galerkin-type series and a modification of the spectral method to turn partial 
differential equations (versus time) into ordinary differential equations for coefficients of the spectral series. Yu and Hickey (2007), Liu et al. (2008), Matsumura et al. (2011), and Occhipinti et al. (2011) performed numerical simulations of AGWs in the atmosphere. Besides direct numerical simulations, propagation and dissipation of mesoscale internal gravity waves generated in the lower atmosphere have recently been studied in a general circulation model (Yi git et al. 2009, 2012) involving a parameterization of gravity wave effects, their nonlinear saturation and dissi-

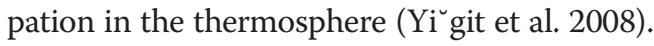

Gavrilov and Kshevetskii (2013a) solved two-dimensional nonlinear AGW equations using a numerical method described by Kshevetskii and Gavrilov (2005) and taking into account fundamental conservation laws. This approach provides the numerical method stability and allows us to consider non-smooth solutions of nonlinear AGW equations. This gave us an opportunity to select generalized physically correct solutions of the equations (Lax 1957; Richtmayer and Morton 1967). Gavrilov and Kshevetskii (2013b, 2014) developed a three-dimensional version of the mentioned above algorithm for simulation of nonlinear AGWs in the atmosphere. They considered propagation of plain sinusoidal wave forcing at the low boundary of the model.

In the present study, we continue a three-dimensional simulation of nonlinear AGWs from the Earth's surface sources into the thermosphere using the algorithm by Gavrilov and Kshevetskii (2013b). We consider AGW forcing by horizontally periodical stationary structures of vertical velocity at the low boundary of the model. Such structures may arise from convection cells transported by background winds. In the present paper, we consider nonlinear wave effects in the middle and upper atmosphere for different amplitudes, horizontal wavelengths, and speeds of the lower boundary wave forcing.

\section{Simulation method}

The three-dimensional numerical model of nonlinear AGWs uses the plain geometry and calculates velocity components $u, v$, and $w$ along horizontal $(x, y)$ and vertical, $z$, axes, respectively. The model also computes deviations of pressure $p^{\prime}$, temperature $T^{\prime}$, and density $\rho^{\prime}$ from stationary values $p_{0}, T_{O}$, and $\rho_{0}$, respectively. The set of nonlinear three-dimensional hydrodynamic equations of the model is described by Gavrilov and Kshevetskii (2013b, 2014) and includes equations of motion, continuity, and heat balance. The upper boundary conditions include zero vertical velocity and zero vertical gradients of horizontal velocity and temperature at altitude $z=500 \mathrm{~km}$. At the Earth's surface, the lower boundary conditions include zero temperature deviations and horizontal velocity components (see Gavrilov and Kshevetskii 2013a,b, 2014). In the present study, we assume periodicity of wave solutions and the lateral boundary conditions in horizontal directions:

$$
f(x, y, z, t)=f\left(x+L_{x}, y+L_{y}, z, t\right)
$$

where $f$ represents any of the simulated hydrodynamic variables, $L_{x}=m \lambda_{x}$ and $L_{y}=n \lambda_{y}$ are the horizontal dimensions of the analyzed atmospheric region, where $m$ and $n$ are integer constants, $\lambda_{x}$ and $\lambda_{y}$ are wavelengths along axes $x$ and $y$, respectively. Variations of vertical velocity $(w)_{z=O}=w(x, y)$ at the lower boundary $z=0$ serve to force the waves in the model.

A three-dimensional numerical scheme of our model is analogous to the two-dimensional algorithm described by Kshevetskii and Gavrilov (2005). It is a revision of the known Lax and Wendroff (1960) scheme. This scheme involves the hydrodynamic conservation laws of momentum, density, and energy. The main mathematical modification of our scheme compared to the classical Lax and Wendroff (1960) one is the implicit approximation of hydrodynamic equations at first time half-step. Kshevetskii (2001a,b,c) showed that the errors from acoustic waves do not increase in time in this case. One more peculiarity of our numerical scheme is the usage of a staggered grid, in which pressure, density, and temperature are specified at the same mesh points and nodes for velocity components $u, v$, and $w$ are shifted half grid spacing along axes $x, y, z$, respectively.

Simulations in this paper use background vertical profiles of $p_{O}, T_{O}$, and $\rho_{O}$ from the model of standard atmosphere MSIS-90 (Hedin 1991) for January at moderate geomagnetic activity (see Figure one in Gavrilov and Kshevetskii 2013b, 2014). We simulate AGWs for different characteristics of the lower boundary forcing. The mean vertical grid spacing is $250 \mathrm{~m}$, but it automatically increases from approximately $0.15 \mathrm{~km}$ in the lower atmosphere up to approximately $1.7 \mathrm{~km}$ at altitude $500 \mathrm{~km}$ because the background and wave fields become more vertically homogeneous at high altitudes. Horizontal grid spacing is $1 / 30$ of horizontal wavelengths $\lambda_{x}$ of the wave forcing (1). Time spacing is determined automatically to provide stability of the numerical equation solution and are 0.3 and $0.9 \mathrm{~s}$ for $\lambda_{x}=60 \mathrm{~km}$ and $\lambda_{x}=200 \mathrm{~km}$, respectively.

Molecular viscosity and heat conductivity do not practically influence long waves in the lower atmosphere, but they are important at higher altitudes. Therefore, our numerical model involves molecular viscosity and heat conductivity. The model does not take into account some physical effects, such as wave dissipation by radiative heat exchange and ion drag, that are of secondary importance for simulating high-frequency atmospheric waves. 


\section{Results of calculations}

Gavrilov and Kshevetskii (2013a,b, 2014) simulated nonlinear AGW propagation from lower boundary forcing having forms of plane waves. In the present study, we assume horizontally periodical distributions of vertical velocity at the Earth's surface of the form of

$$
(w)_{z=0}=W_{0} \cos \left[k_{x}\left(x-c_{x} t\right)\right] \cos \left[k_{y}\left(y-c_{y} t\right)\right] \text { at } t>0,
$$

where $k_{x}=2 \pi / \lambda_{x}, k_{y}=2 \pi / \lambda_{y}$ and $c_{x}, c_{y}$ are wavenumbers and phase speeds along horizontal axes $x$ and $y$, respectively; $W_{O}$ is the maximum wave forcing amplitude. Equation 2 represents horizontally moving periodical structures of vertical velocity at the bottom boundary. Medvedev and Gavrilov (1995) analyzed AGW generation by nonlinear interactions caused by meteorological and turbulent motions in the atmosphere. They found that such interactions could produce components of wave spectra having broad variety of amplitudes, wavelengths, and other parameters. The forcing (2) may approximate spectral components of convective and turbulent AGW sources transported by the mean winds in the atmosphere (Townsend 1965, 1966). In the present paper, we present calculations for waves with $c_{x}=30 \mathrm{~m} / \mathrm{s}$ and $c_{x}=$ $100 \mathrm{~m} / \mathrm{s}$ at $c_{y}=0$ and the same period $\tau=2 \times 10^{3} \mathrm{~s}$. In this case, (2) is equivalent to propagating waves along the horizontal axis $x$ with stationary sinusoidal amplitude change along the axis $y$. The described numerical simulations were performed starting from the MSIS means state (zero perturbations) at the moment $t=0$ of activating the wave forcing at the lower boundary.

The wave source (2) activating at $t=0$ contains a Heaviside step function in time and a $\delta$-function distribution in vertical direction with the peak at $z=0$. At the beginning, Fourier transforms of such abrupt functions contain wide spectra of components covering all possible ranges of frequencies and vertical wavenumbers (e.g., Weisstein 2014). Then the wave source spectrum has the main peak at frequency $k_{x} c_{x}$. Figure 1 shows examples of frequency spectra (in relative units) of the wave forcing (2) calculated using sliding intervals at different times $t$ after activating the wave forcing. The spectra are very broad in the beginning and become narrower with time.

After activating the wave forcing (2), faster acoustic and extremely long gravity wave modes will quickly reach high altitudes. Calculations show that in the horizontally periodic case (1), these perturbations in a few minutes can arrive at altitudes of $100 \mathrm{~km}$ and above and form quasivertical wave fronts similar to those in Figure 1a,b,c of the paper by Gavrilov and Kshevetskii (2013a). These first waves dissipate due to molecular viscosity and thermal conduction. As time increases, progressively more of the larger vertical wavelength waves are removed by

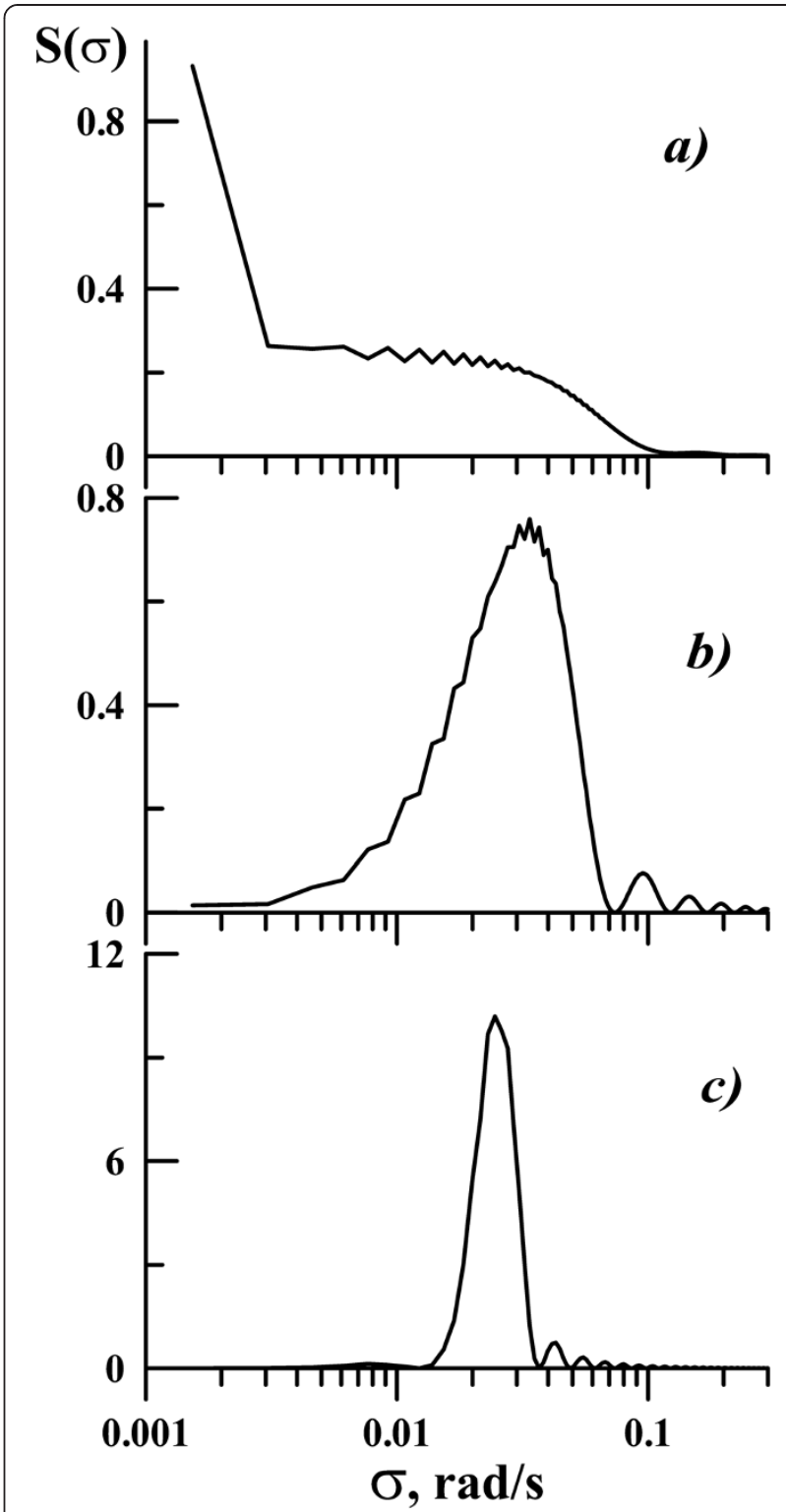

Figure 1 Frequency spectra (in relative units) of the wave forcing (2) at different times after activation. $t=0.25 \tau$ (a), $t=\tau$ (b), and $t=4 \tau$ (c).

dissipation, implying that vertical wavelengths at a given altitude in the middle atmosphere should decrease with increasing time (Heale et al. 2014). After a certain transition time, initial acoustic and gravity wave modes disperse and the vertical structure corresponds to the basic spectral component of the surface forcing (2) having horizontal phase speed $c_{x}$ and horizontal wave number $k_{x}$.

Figures 2 and 3 reveal wave fields of vertical and horizontal velocities, respectively, for different instances $t$ after activating the wave forcing (2) with different $c_{x}$ and $\lambda_{x}$. One can see the surfaces of constant phases inclined to the horizon below altitudes about $100 \mathrm{~km}$. A theory of plane linear stationary AGWs (e.g., Gossard and Hooke 


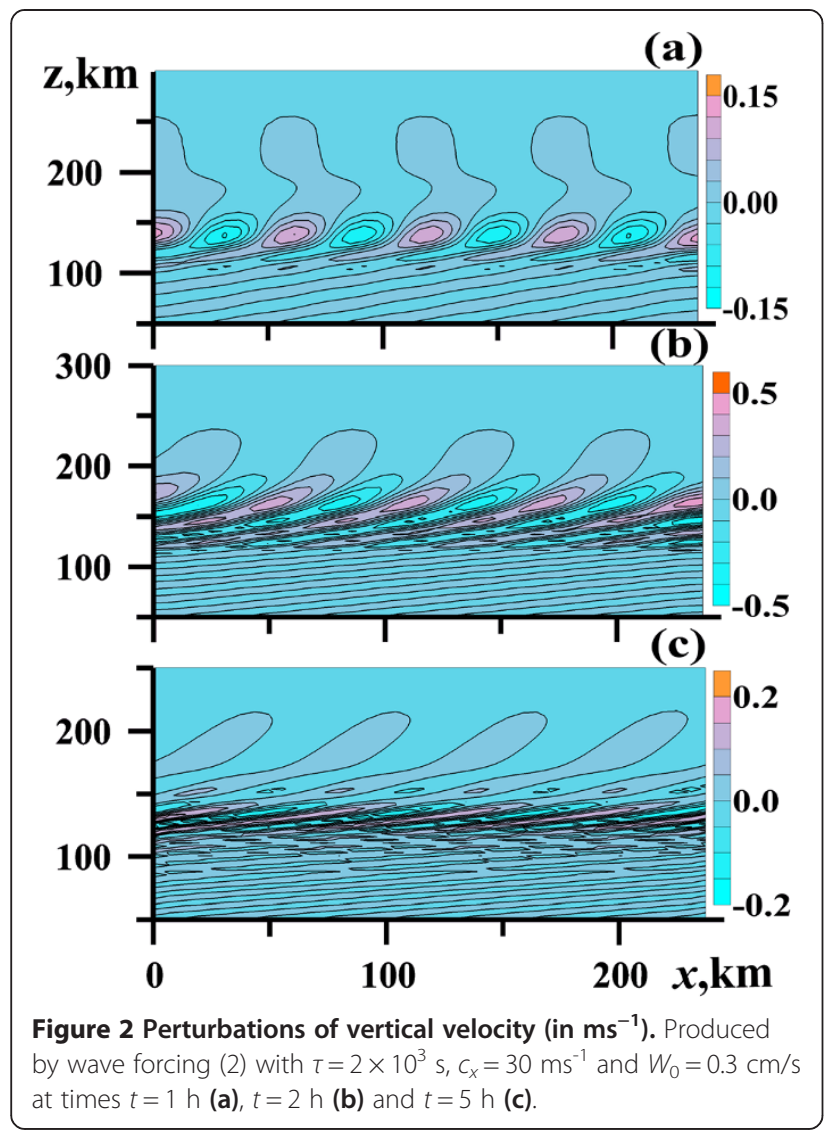

1975) gives the angle of this inclination to be $\alpha \sim \operatorname{arsin}$ $\left(\tau_{N} / \tau\right)$, where $\tau_{N}$ and $\tau$ are the Brunt-Vaisala and AGW periods, respectively. This corresponds to the theoretical vertical wavelength $\lambda_{z} \approx c_{x} \tau_{N} \sim 9$ to $12 \mathrm{~km}$ for $c_{x}=30 \mathrm{~ms}^{-1}$ and $\lambda_{z} \sim 30$ to $40 \mathrm{~km}$ for $c_{x}=100 \mathrm{~ms}^{-1}$ at typical $\tau_{N} \sim(3$ to 4) $110^{2} \mathrm{~s}$ at altitudes 50 to $100 \mathrm{~km}$ in January.

One can estimate simulated effective $\lambda_{z}$ values as average vertical distances between inclined quasi-linear wave fronts in the lower parts of Figures 2a,b,c and 3a,b,c. This gives $\lambda_{z} \approx 50,14,10 \mathrm{~km}$ for $c_{x}=30 \mathrm{~ms}^{-1}$ at altitudes 50 to $100 \mathrm{~km}$ in Figure 2a,b,c, respectively, for the time moments $t=1,2$, and $5 \mathrm{~h}$ after activating the lower boundary wave source (2) in the model. Similar estimations for the fields of horizontal velocity in Figure 3a,b,c give, respectively, $\lambda_{z} \approx 80,55,45 \mathrm{~km}$ at $t=1,2,5 \mathrm{~h}$ for $c_{x}=100 \mathrm{~ms}^{-1}$ at altitudes 50 to $100 \mathrm{~km}$. One can see that in both cases, $\lambda_{z}$ decreases in time (as described before) and tends to the theoretically predicted values (see above) after a transition periods, which can last more than ten periods of the wave forcing $\tau=\lambda_{x} / c_{x}=2 \times 10^{3} \mathrm{~s}$ for both Figures 2 and 3. Above altitudes 150 to $200 \mathrm{~km}$, wave front angles $\alpha$ increase in Figures 2 and 3 due to increasing the vertical wavelength, molecular viscosity, and heat conduction.

Increases in AGW amplitudes can lead to instabilities of wave velocity and temperature fields. Kshevetskii and Gavrilov (2005) found that dynamical instabilities of the

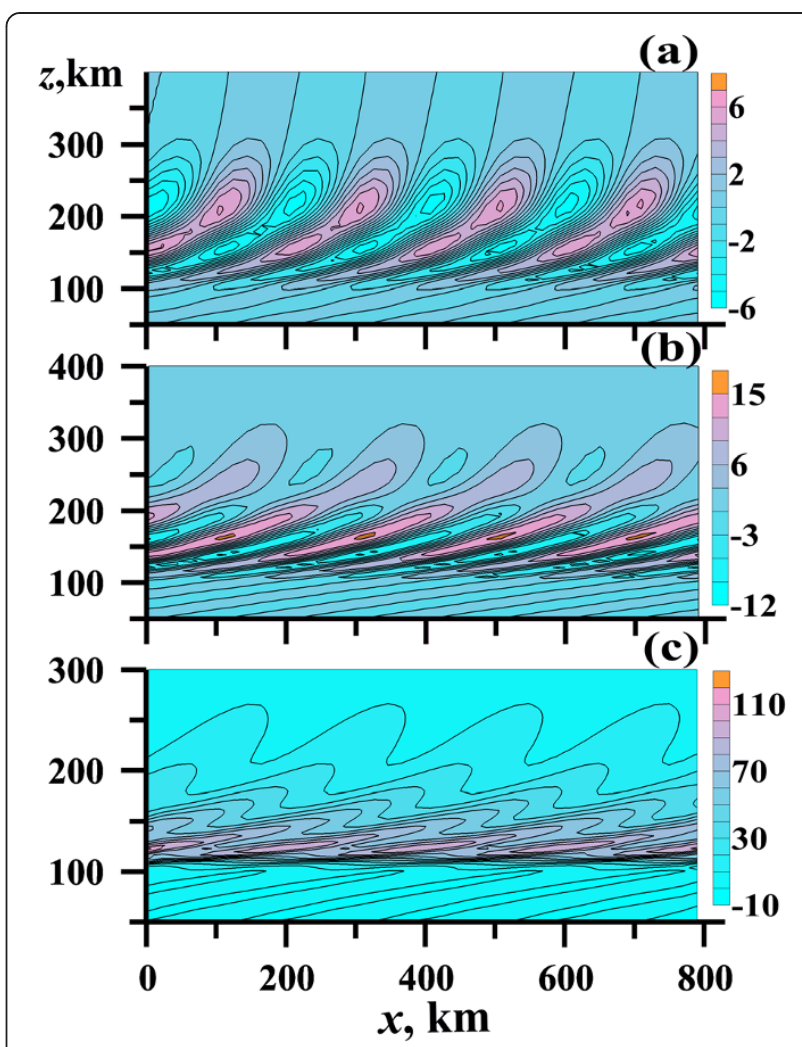

Figure 3 Perturbations of horizontal velocity (in $\mathrm{ms}^{-1}$ ). Produced by wave forcing (2) with $\tau=2 \times 10^{3} \mathrm{~s}, c_{x}=100 \mathrm{~ms}^{-1}$ and $W_{0}=0.3 \mathrm{~cm} / \mathrm{s}$ at times $t=1 \mathrm{~h}(\mathbf{a}), t=2 \mathrm{~h}$ (b) and $t=5 \mathrm{~h}$ (c).

wind shears could be the main reason of AGW breaking at moderate amplitudes of the wave forcing. Wave breaking at large forcing amplitudes could be due to convective instabilities of temperature wave profiles. In Figures 2 and 3 above altitude $100 \mathrm{~km}$, one can see small-scale structures produced by AGW instabilities.

Dissipation of nonlinear breaking AGWs may produce accelerations of the mean flow. These accelerations are important for the development of general circulation of the middle atmosphere (e.g., Lindzen 1981). Accelerations produced by AGWs may develop mean flows at altitudes of substantial dissipation of the propagating waves. In Figure 3c, one can see such increase in the mean horizontal velocity above altitudes 110 to $120 \mathrm{~km}$. Modifications of the mean horizontal flows by dissipating AGWs are known effects (e.g., Fritts and Alexander 2003). Other models of nonlinear AGWs also demonstrated such mean flow changes (e.g., Liu et al. 2008).

Figure 4 represents distributions of vertical velocity in horizontal planes at different altitudes for AGW with $c_{x}=100 \mathrm{~ms}^{-1}$ at time $t=5 \mathrm{~h}$ after activating the forcing (2). At all altitudes in Figure 4, one can see structures of maxima and minima very similar to the structure of the lower boundary wave forcing described by (2). At low wave forcing, analyses performed for AGWs with different 


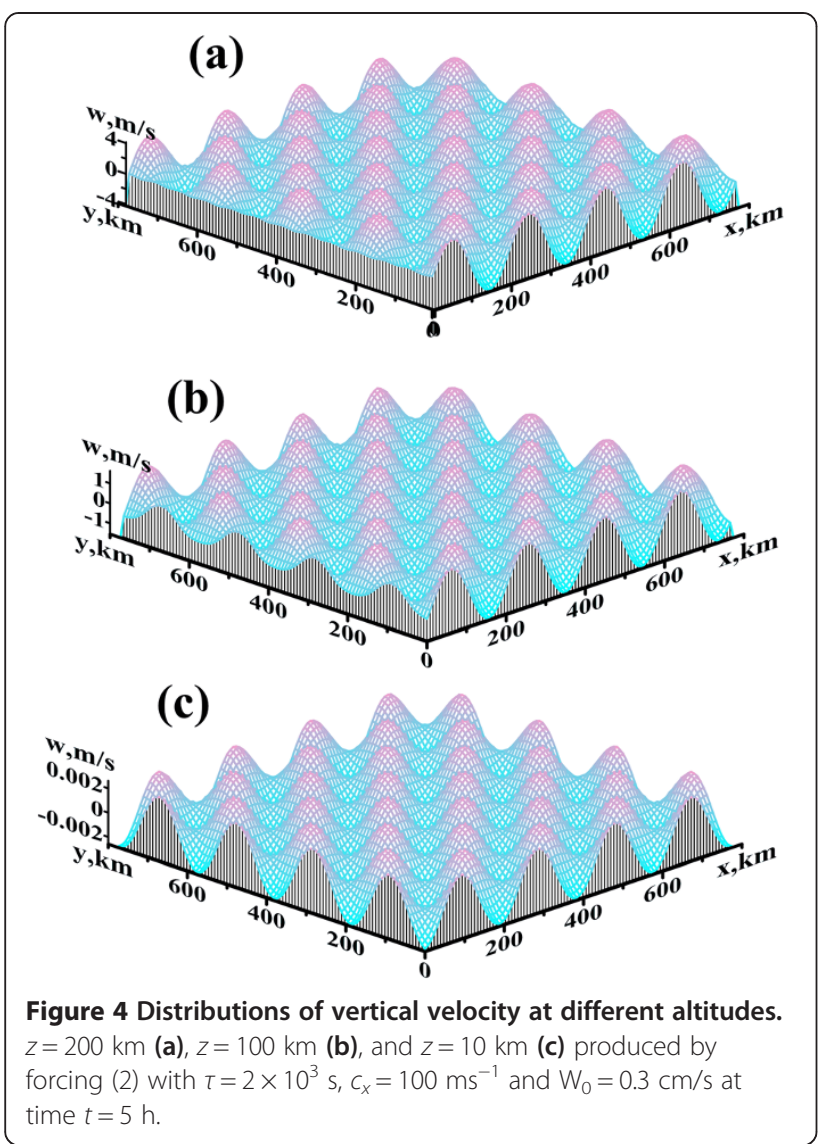

$\lambda_{x}$ and $c_{x}$ and for different wave fields reveal in all cases similarities between the horizontal distributions of wave fields at higher altitudes and the distribution of surface wave source (2). The only differences in horizontal structures at individual altitudes are different amplitudes and phase shifts of the wave fields. This shows that when atmospheric wave sources have amplitude changes in horizontal directions, AGWs may transport these structures to the other altitudes up to very high levels.

Figure 5 shows simulated dependences of standard deviations of vertical velocity on time after wave forcing activation in horizontal planes located at different altitudes. Figure 5 shows that the standard deviations grow during transition intervals after the wave source activation and then tend to constant values different at each altitude. In the horizontally periodical case (1), these standard deviations tend to be a half of AGW amplitudes at large $t$, when the wave process becomes quasi-stationary. Vertical group velocity of a plane AGW spectral component is $c_{g z} \approx \lambda_{z} / \tau$, and the time of their energy arrival to altitude $z$ is $t_{e z}=z / c_{g z}$. For the main considered spectral components of excitation (2) with $\tau=2 \times 10^{3} \mathrm{~s}$ and average values $\lambda_{z} \sim 35 \mathrm{~km}$ for $c_{x}=100 \mathrm{~ms}^{-1}$ and $\lambda_{z} \sim 10 \mathrm{~km}$ for $c_{x}=30 \mathrm{~ms}^{-1}$ (see above), we can obtain $t_{e z} / \tau=z / \lambda_{z} \sim 0.3,1.7,2.9$ and $t_{e z} / \tau \sim 1,6,10$ at altitudes 10,60 , and $100 \mathrm{~km}$ for both $c_{x}$, respectively.

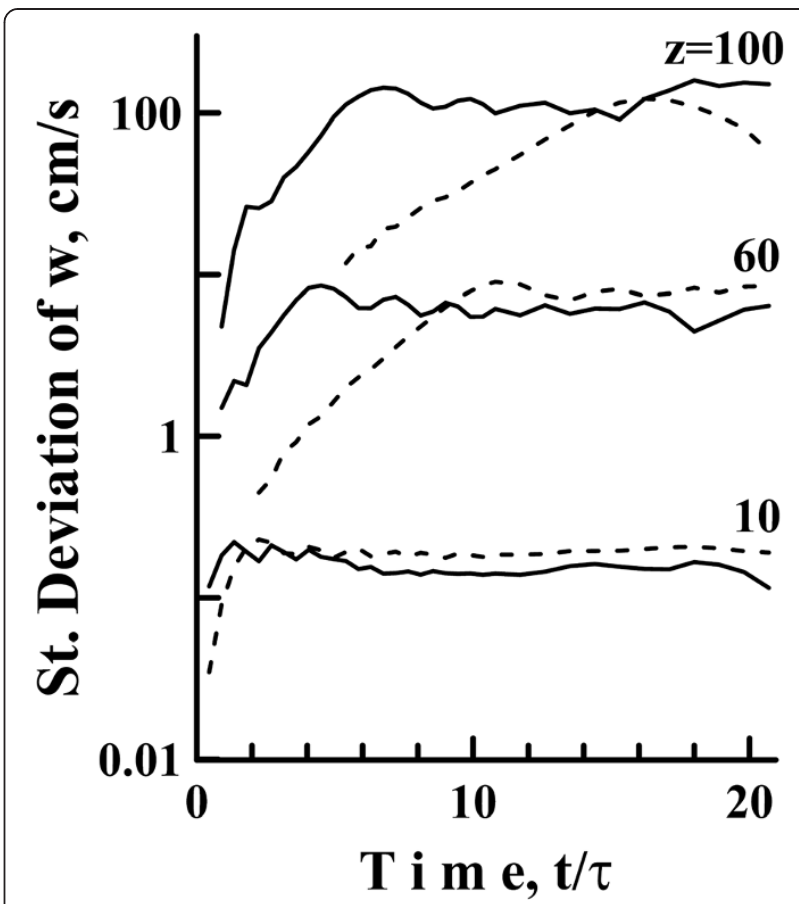

Figure $\mathbf{5}$ Standard deviations of vertical velocity. At altitudes $z=10,60$, and $100 \mathrm{~km}$ versus time for AGWs with $c_{x}=100 \mathrm{~m} / \mathrm{s}$ (solid lines), and $c_{x}=30 \mathrm{~m} / \mathrm{s}$ (dashed lines) for $\tau=2 \times 10^{3} \mathrm{~s}$ and $W_{0}=0.3 \mathrm{~cm} / \mathrm{s}$.

Consideration of Figure 5 reveals times $t_{m}$ of the first maxima of standard deviations at altitudes $10,60,100 \mathrm{~km}$ (after which their variations become smaller) to be $t_{m} / \tau \sim 1.3,4.5,6.8$ for $c_{x}=100 \mathrm{~m} / \mathrm{s}$ and $t_{m} / \tau \sim 2.3,10.8$, 16.2 for $c_{x}=30 \mathrm{~m} / \mathrm{s}$, respectively. These values of $t_{m} / \tau$ are 1.6 to 4 times larger than respective $t_{e z} / \tau$. Therefore, durations of the transition intervals are larger for smaller $c_{x}$. These durations grow with height and may exceed ten wave periods at altitude $100 \mathrm{~km}$.

Figure 5 shows that after reaching the maximum at a given altitude, the standard deviation is not constant, but makes small oscillations around slowly varying values. These oscillations could be caused by partial reflections of wave energy from vertical inhomogeneities of temperature and molecular viscosity and heat conduction in regions of their rapid changes in the middle and upper atmosphere. In Figure 5, these oscillations are larger for solid lines showing that partial reflections could be larger for wave modes with larger $c_{x}$ and longer $\lambda_{z}$. Partial reflections taking into account in the three-dimensional numerical model can also be a reason for smaller quasi-stationary standard deviations corresponding to $c_{x}=100 \mathrm{~m} / \mathrm{s}$ at $z=$ $10,60 \mathrm{~km}$ compared to that for $c_{x}=30 \mathrm{~m} / \mathrm{s}$ in Figure 5 .

Figures 6 and 7 depict examples of vertical profiles of vertical and horizontal velocities at different times for AGW excitations (2) with $c_{x}=30 \mathrm{~ms}^{-1}$ and $c_{x}=100 \mathrm{~ms}^{-1}$, respectively. Comparison of Figures $6 \mathrm{a}$ and $7 \mathrm{a}$ with 


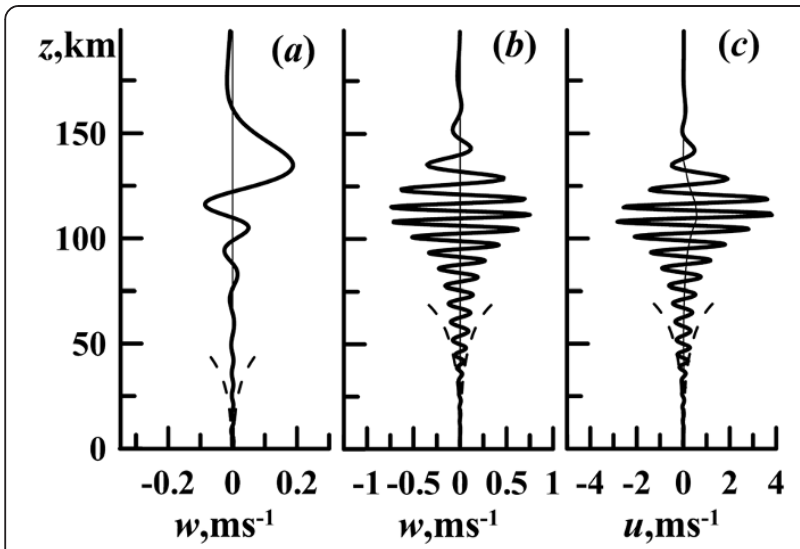

Figure 6 Vertical profiles at fixed $x=2 \mathrm{~km}$ and $y=188 \mathrm{~km}$ of vertical velocity. At $t=1 \mathrm{~h}(\mathbf{a}), t=5 \mathrm{~h}(\mathbf{b})$, and horizontal velocity at $t=5 \mathrm{~h} \mathrm{(c)} \mathrm{produced} \mathrm{by} \mathrm{wave} \mathrm{forcing} \mathrm{(2)} \mathrm{with} \tau=2 \times 10^{3} \mathrm{~s}, \mathrm{c}_{x}=30 \mathrm{~ms}^{-1}$ and $W_{0}=0.3 \mathrm{~cm} / \mathrm{s}$. Thin solid lines show respective mean values. Dashed lines reveal dependences proportional to $\pm\left[\rho_{0}(0) / \rho_{0}(\mathrm{z})\right]^{1 / 2}$.

Figures $6 \mathrm{~b}$ and $7 \mathrm{~b}$ for $t=1 \mathrm{~h}$ and $t=5 \mathrm{~h}$ shows larger vertical wavelength at earlier times as it was found in Figure 2 above. The longer vertical wavelengths at initial moments are formed by harmonics with faster horizontal and vertical group velocities. After a certain time, they disperse leaving the vertical structure corresponding to the preset forcing at the lower boundary (see discussion above). AGW amplitudes below $150 \mathrm{~km}$ are smaller in Figures $6 \mathrm{a}$ and $7 \mathrm{a}$ for earlier time instance, than respective amplitudes for later time in Figures $6 \mathrm{~b}$ and 7b. Dashed lines in Figures 6 and 7 correspond to exponential amplitude growth proportional to $\left[\rho_{0}(0) / \rho_{0}(\mathrm{z})\right]^{1 / 2}$ and predicted by the theory of stationary nondissipative linear AGWs. One can see that calculated AGW amplitudes have better match to exponential curve in Figures $6 \mathrm{~b}$ and $7 \mathrm{~b}$ than that in Figure $6 \mathrm{a}$ and $7 \mathrm{a}$ due to amplitudes growth in time. Amplitude vertical growth rates slower

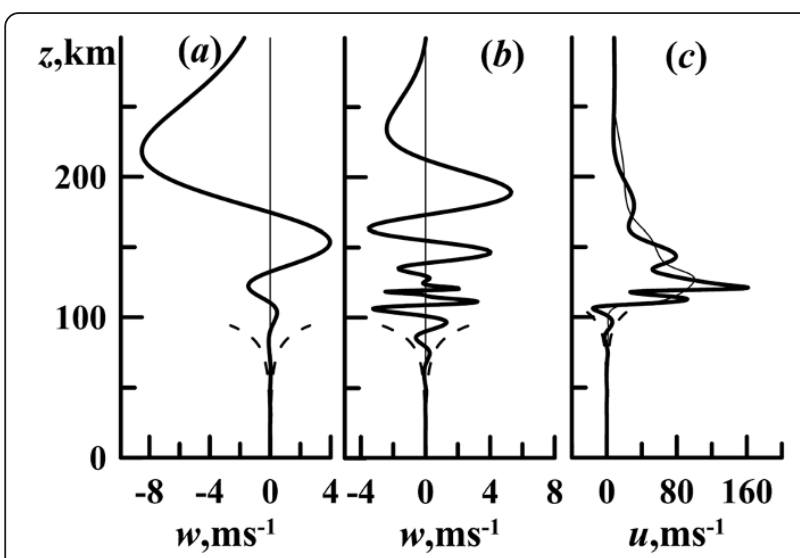

Figure 7 Same as Figure 6, but for the wave with horizontal phase speed $c_{\boldsymbol{x}}=100 \mathrm{~ms}^{-1}$. Vertical velocity at $t=1 \mathrm{~h}(\mathrm{a}), t=5 \mathrm{~h}$ (b), and horizontal velocity at $t=5 \mathrm{~h}$ (c). than the exponential one are frequently treated as evidence of AGW dissipation and saturation (e.g., Fritts and Alexander 2003). Figures 6 and 7 show that one more reason for slower amplitude growth could be transition processes in wave fields, during which the discrepancy between simulated and exponential amplitude growth rate is larger at $t=1 \mathrm{~h}$ than that at $t=5 \mathrm{~h}$ even for the same AGW at lower altitudes. Figures $6 \mathrm{~b}, \mathrm{c}$ and $7 \mathrm{~b}, \mathrm{c}$ show that at $t=5 \mathrm{~h}$, wave amplitudes mach to the exponential growth up to altitudes 50 to $60 \mathrm{~km}$ for AGW mode with $c_{x}=30 \mathrm{~m} / \mathrm{s}$ and up to 70 to $80 \mathrm{~km}$ for $c_{x}=100 \mathrm{~m} / \mathrm{s}$. This could be explained by larger dissipation and slower vertical propagation of AGWs with smaller $c_{x}$ due to their shorter vertical wavelengths. Additional reasons for slow down vertical amplitude growth of primary AGW in nonlinear model can be generation of secondary wave modes and the mean flow.

Figures $6 \mathrm{c}$ and $7 \mathrm{c}$ show vertical profiles of horizontal velocity $u$ at $t=5 \mathrm{~h}$. These profiles are similar to the respective profiles of vertical velocity in Figures $6 \mathrm{~b}$ and $7 \mathrm{~b}$, but are shifted to positive values due to generation of the wave-induced jet streams above $100 \mathrm{~km}$ (see also Figure 2c) in the directions of AGW horizontal propagation. Corresponding horizontal mean velocities shown with thin solid lines in Figure $7 \mathrm{c}$ are larger for AGW with $c_{x}=100 \mathrm{~m} / \mathrm{s}$ than that in Figure $6 \mathrm{c}$. The upper limit for horizontal velocity in the wave-induced jet stream is $c_{x}$ (Kshevetskii and Gavrilov 2005) and it is larger for faster waves.

Vertical component of the wave flux of horizontal momentum $F_{m z}=\rho_{0}\left\langle u^{\prime} w^{\prime}\right\rangle \sim \rho_{O} \tau_{N} W^{2} /(2 \tau)$, where $<>$ denotes averaging over the wave period. As far as periods and amplitudes at the bottom boundary are the same for both considered AGW modes, they have there the same $F_{m z} \sim 3.3 \times 10^{-5} \mathrm{~kg} \mathrm{~m}^{-1} \mathrm{~s}^{-2}$ at horizontal coordinates $y$ corresponding to maximum wave excitations (2). Calculations show that $F_{m z}$ does not almost change up to altitudes 50 to $60 \mathrm{~km}$ for AGW mode with $c_{x}=30 \mathrm{~m} / \mathrm{s}$ and up to 70 to $80 \mathrm{~km}$ for $c_{x}=100 \mathrm{~m} / \mathrm{s}$, then decreases due to wave dissipation. Therefore, the amplitude of longer vertical wavelength mode with $c_{x}=100 \mathrm{~m} / \mathrm{s}$ grows up to higher altitudes (see above) and substantially exceeds the amplitude of the mode with $c_{x}=30 \mathrm{~m} / \mathrm{s}$ near $100 \mathrm{~km}$ (compare Figures 6 and 7). This may explain why wave mode with $c_{x}=100 \mathrm{~m} / \mathrm{s}$ produces larger dynamical effects and stronger waveinduced jet at high altitudes than the mode with smaller $c_{x}$.

Increased mean flows combined with superimposed wave structures (see Figure 7c) may produce narrow layers with large vertical wind shears. Dynamical instabilities in these layers can increase AGW dissipation and wave accelerations of the mean flow leading to faster growing of the wave-induced jet stream. Such 'self-accelerating' process produces quite narrow peaks of horizontal velocity with a maximum larger than $150 \mathrm{~ms}^{-1}$ in Figure 7c. Substantial energy transition from AGW to the mean flow 
and changes of propagation conditions decreases wave amplitudes and vertical wavelengths at altitudes of the jet 100 to $150 \mathrm{~km}$ in Figure 7b,c.

Larsen (2000) and Larsen et al. (2005) reported about intensive jet streams frequently observed at altitudes near $100 \mathrm{~km}$. Several mechanisms were proposed to explain these jets. Mentioned above self-accelerating growth of the mean flow produced by fast AGWs and their superposition with wave fields may be one of the mechanisms for formation of strong narrow peaks of horizontal speed in the upper atmosphere. Figure $7 \mathrm{c}$ shows that times of only a few wave periods may be enough for such peaks creation by fast AGWs even at moderate amplitudes of the low-boundary wave forcing in the numerical model.

Figure 5 shows quite long transition time intervals of getting developed structures of stationary AGWs after activating the lower boundary wave excitation (2). Average characteristics of the wave fields change during these transition intervals. Observations (e.g., Fritts and Alexander 2003) frequently reveal relatively short pulses of a few AGW wavelengths and periods in the atmosphere. This shows that durations of many AGW sources in the atmosphere are relatively short (e.g., Fritts et al. 2006). Respective AGW pulses may have not enough time for becoming stationary during the activity of the wave sources.

Therefore, we may assume that a substantial proportion of AGWs observed in the atmosphere may be nonstationary. Hence, a question may arise about the applicability of the steady wave approximation to the real atmosphere. Such approximation is commonly used in parameterizations of gravity wave effects in the atmosphere (e.g., Gossard and Hooke 1975) in applications to non-stationary AGWs. Further observations and modeling are required for better understanding the wave propagation and resulting perturbations produced by variable wave sources in the atmosphere.

\section{Conclusion}

Numerical simulations of nonlinear breaking AGW propagation to the middle and upper atmosphere from a horizontally periodical wave forcing near the ground were made. After activating the tropospheric wave forcing, atmospheric waves in a few minutes can propagate to altitudes above $100 \mathrm{~km}$. Initially quasi-vertical surfaces of constant phases become inclined to the horizon below altitudes about $100 \mathrm{~km}$ after some transition time intervals. Vertical wavelengths decrease and tend to theoretically predicted values after times longer than several periods of the wave forcing. Decreases in vertical wavelengths and increases in AGW amplitudes can lead to wave instabilities, accelerations of the mean flow and wave-induced jet streams at altitudes above $100 \mathrm{~km}$. AGWs may transport horizontal amplitude modulation of atmospheric wave sources to the other altitudes up to very high levels. One of the reasons for AGW amplitude growth rate slower than the non-dissipative exponential grows could be low wave amplitudes in the beginning of the transition processes after activation of atmospheric wave sources. Production of wave-induced mean jets and their superposition with nonlinear unstable dissipative AGWs may be one of mechanisms for formation of strong narrow peaks of horizontal speed in the upper atmosphere. Due to substantial transition times, many of AGW pulses in the atmosphere may have not enough time for becoming stationary during the activity of wave sources. Further observations and modeling are necessary for better understanding nonlinear AGW behavior in the atmosphere.

\section{Competing interests}

The authors declare that they have no competing interests.

\section{Authors' contributions}

NG made computer calculations and plots and their analysis. SK developed the numerical model and participated in the analysis of the results. Both authors read and approved the final manuscript.

\section{Acknowledgements}

This work was partly supported by the Russian Basic Research Foundation and by the Russian Scientific Foundation (grant 14-17-00685). The authors thank A. N. Gavrilov for help in improving computer codes.

Received: 22 January 2014 Accepted: 22 July 2014

Published: 6 August 2014

\section{References}

Andreassen O, Hvidsten O, Fritts D, Arendt S (1998) Vorticity dynamics in a breaking internal gravity wave. Part 1. Initial instability evolution. J Fluid Mech 367:27-46

Baker D, Schubert G (2000) Convectively generated internal gravity waves in the lower atmosphere of Venus. Part II: mean wind shear and wave-mean flow interaction. J Atmos Sci 57:200-215

Fritts DC, Garten JF (1996) Wave breaking and transition to turbulence in stratified shear flows. J Atmos Sci 53(8):1057-1085

Fritts DC, Alexander MJ (2003) Gravity wave dynamics and effects in the middle atmosphere. Rev Geophys 41(1):1003, doi:10.1029/2001RG000106

Fritts DC, Vadas SL, Wan K, Werne JA (2006) Mean and variable forcing of the middle atmosphere by gravity waves. J Atmos Solar-Terr Phys 68:247-265

Fritts DC, Wang L, Werne J, Lund T, Wan K (2009) Gravity wave instability dynamics at high Reynolds numbers. Part II: turbulence evolution, structure, and anisotropy. J Atmos Sci 66:1149-1171

Fritts DC, Franke PM, Wan K, Lund T, Werne J (2011) Computation of clear-air radar backscatter from numerical simulations of turbulence: 2 . Backscatter moments throughout the lifecycle of a Kelvin-Helmholtz instability. J Geophys Res 116, D11105

Gavrilov NM (2007) Structure of the mesoscale variability of the troposphere and stratosphere found from radio refraction measurements via CHAMP satellite. Izvestia Atmos Oceanic Phys 43(4):451-460

Gavrilov NM, Fukao S (1999) A comparison of seasonal variations of gravity wave intensity observed by the MU radar with a theoretical model. J Atmos Sci 56:3485-3494

Gavrilov NM, Kshevetskii SP (2013a) Numerical modeling of propagation of breaking nonlinear acoustic-gravity waves from the lower to the upper atmosphere. Adv Space Res 51(7):1168-1174

Gavrilov NM, Kshevetskii SP (2013b) Study of acoustic-gravity wave propagation in the middle and upper atmosphere using numerical modeling. Chem Phys 32(12):65-72 (In Russian)

Gavrilov NM, Kshevetskii SP (2014) Numerical modeling of the propagation of nonlinear acoustic-gravity waves in the middle and upper atmosphere. Izvestiya, Atmos Oceanic Phys 50(1):66-72

Gossard EE, Hooke WH (1975) Waves in the atmosphere. Elsevier Sci Publ Co, Amsterdam-Oxford-New York 
Heale CJ, Snively J, Hickey MP, Ali C (2014) Thermospheric dissipation of upward propagating gravity wave packets. J Geophys Res 119(5A):357-387, doi:10.1002/2013JA019387

Hedin AE (1991) Neutral atmosphere empirical model from the surface to lower exosphere MSISE-90, extension of the MSIS thermosphere model into the middle and lower atmosphere. J Geophys Res 96:1159-1172

Kshevetskii SP (2001a) Modelling of propagation of internal gravity waves in gases. Comput Math and Math Phys 41(2):295-310

Kshevetskii SP (2001b) Analytical and numerical investigation of nonlinear internal gravity waves. Nonlin Proc Geophys 8:37-53

Kshevetskii SP (2001c) Numerical simulation of nonlinear internal gravity waves. Comput Math and Math Phys 41(12):1777-1791

Kshevetskii SP, Gavrilov NM (2005) Vertical propagation, breaking and effects of nonlinear gravity waves in the atmosphere. J Atmos Solar-Terr Phys 67:1014-1030

Larsen MF (2000) A shear instability seeding mechanism for quasiperiodic radar echoes. J Geophys Res 105:24931-24940

Larsen MF, Yamamoto M, Fukao S, Tsunoda RT, Saito A (2005) Observations of neutral winds, wind shears, and wave structure during a sporadic- E/QP event. Ann Geophys 23:2369-2375, doi:1432-0576/ ag/2005-23-2369

Lax PD (1957) Hyperbolic systems of conservation laws. Comm Pure Appl Math 10:537-566

Lax PD, Wendroff B (1960) Hyperbolic systems of conservation laws. Comm Pure Appl Math 13:217-237

Lindzen RS (1981) Turbulence and stress owing to gravity wave and tidal breakdown. J Geophys Res 86:9707-9714

Liu X, Xu J, Liu H-L, Ma R (2008) Nonlinear interactions between gravity waves with different wavelengths and diurnal tide. J Geophys Res 113, D08112, doi:10.1029/2007JD009136

Matsumura M, Saito A, Iyemori T, Shinagawa H, Tsugawa T, Otsuka Y, Nishioka M, Chen $\mathrm{CH}$ (2011) Numerical simulations of atmospheric waves excited by the 2011 off the Pacific coast of Tohoku Earthquake. Earth Planets Space 63(7):885-889, doi:10.5047/eps.2011.07.015

Medvedev AS, Gavrilov NM (1995) The nonlinear mechanism of gravity wave generation by meteorological motions in the atmosphere. J Atmos Terr Phys 57:1221-1231

Occhipinti G, Coïsson P, Makela JJ, Allgeyer S, Kherani A, Hébert H, Lognonné P (2011) Three-dimensional numerical modeling of tsunami-related internal gravity waves in the Hawaiian atmosphere. Earth Planets Space 63(7):847-851, doi:10.5047/eps.2011.06.051

Ogawa T, Miyoshi Y, Otsuka Y, Nakamura T, Shiokawa K (2009) Equatorial GPS ionospheric scintillations over Kototabang, Indonesia and their relation to atmospheric waves from below. Earth Planets Space 61(4):397-410

Ogawa T, Nishitani N, Tsugawa T, Shiokawa K (2013) Giant ionospheric disturbances observed with the SuperDARN Hokkaido HF radar and GPS network after the 2011 Tohoku earthquake. Earth Planets Space 64:1295-1307, doi:10.5047/eps.2012.08.001

Richtmayer RR, Morton KW (1967) Difference methods for initial-value problems. Intersci Publ, New York

Shiokawa K, Otsuka Y, Ogawa T (2009) Propagation characteristics of nighttime mesospheric and thermospheric waves observed by optical mesosphere thermosphere imagers at middle and low latitudes. Earth Planets Space 61(4):479-491

Suzuki S, Shiokawa K, Otsuka Y, Ogawa T, Kubota M, Tsutsumi M, Nakamura T, David C, Fritts DC (2007) Gravity wave momentum flux in the upper mesosphere derived from $\mathrm{OH}$ airglow imaging measurements. Earth Planets Space 59(5):421-428

Townsend AA (1965) Excitation of internal waves by a turbulent boundary layer. J Fluid Mech 22:241-252

Townsend AA (1966) Internal waves produced by a convective layer. J Fluid Mech 24:307-319

Weisstein EW (2014) MathWorld - a Wolfram web resource. http://mathworld. wolfram.com/HeavisideStepFunction.html; http://mathworld.wolfram. com/DeltaFunction.html

Yi git E, Aylward AD, Medvedev AS (2008) Parameterization of the effects of vertically propagating gravity waves for thermosphere general circulation models: sensitivity study. J Geophys Res 113:D19106, doi:10.1029/2008JD010135

Yi git E, Medvedev AS, Aylward AD, Hartogh P, Harris MJ (2009) Modeling the effects of gravity wave momentum deposition on the general circulation above the turbopause. J Geophys Res 114, D07101, doi:10.1029/2008JD011132
Yi git E, Medvedev AS, Aylward AD, Ridley AJ, Harris MJ, Moldwin MB, Hartogh P (2012) Dynamical effects of internal gravity waves in the equinoctial thermosphere. J Atmos Sol-Terr Phys, doi:10.1016/j.jastp.2011.11.014

Yu Y, Hickey MP (2007) Numerical modeling of a gravity wave packet ducted by the thermal structure of the atmosphere. J Geophys Res 112, A06308, doi:10.1029/2006JA012092

doi:10.1186/1880-5981-66-88

Cite this article as: Gavrilov and Kshevetskii: Three-dimensional numerical simulation of nonlinear acoustic-gravity wave propagation from the troposphere to the thermosphere. Earth, Planets and Space 2014 66:88.

\section{Submit your manuscript to a SpringerOpen ${ }^{\odot}$ journal and benefit from:}

- Convenient online submission

- Rigorous peer review

- Immediate publication on acceptance

- Open access: articles freely available online

- High visibility within the field

- Retaining the copyright to your article

Submit your next manuscript at $>$ springeropen.com 